\title{
Trends in population attributable fraction of acute coronary syndrome and ischaemic stroke due to diabetes in Finland
}

\author{
K. Winell • A. Pietilä • M. Niemi • A. Reunanen • \\ V. Salomaa
}

Received: 2 November 2010 /Accepted: 13 June 2011 / Published online: 3 August 2011

(C) Springer-Verlag 2011

\begin{abstract}
Aims/hypothesis Cardiovascular disease (CVD) event rates are decreasing, but the prevalence of diabetes is increasing. The effect of these developments on the population attributable fraction (PAF) of CVD events due to diabetes is not known.

Methods We used country-wide healthcare registers to identify all persons aged $25-80$ years treated for diabetes in Finland during 1992-2002. These data were further linked to the National Cardiovascular Disease Register to identify the first CVD events (acute coronary syndrome and ischaemic stroke) among the individuals with and without diabetes. We calculated the annual PAF of the first CVD events due to diabetes separately for men and women.

Results The number of men treated for diabetes each year almost doubled during the study period from 37,073 to 69,158 between 1992 and 2002. Among women, the number increased from 42,485 to 57,372 . The annual number of first CVD events in the country declined among men with diabetes from 13,436 to 12,678 and among women with diabetes from 8,658 to 7,521 between 1992 and 2002. During the same period, the PAF due to diabetes of the first CVD events increased among men from $11.4 \%$ (95\% CI 10.8, 12.0\%) to $13.8 \%$ (95\% CI 13.2, 14.5\%) and decreased among women from $20.1 \%$ (95\% CI $19.2,21.0 \%$ ) to $16.9 \%$ (95\% CI 15.9 , $17.8 \%)$. The trends in PAF were different between the sexes $(p<0.001$ for the interaction year $\times$ sex $)$.
\end{abstract}

Electronic supplementary material The online version of this article (doi:10.1007/s00125-011-2262-x) contains peer-reviewed but unedited supplementary material, which is available to authorised users.

K. Winell $(\bowtie) \cdot$ A. Pietilä $\cdot$ M. Niemi $\cdot$ A. Reunanen $\cdot$ V. Salomaa National Institute for Health and Welfare,

PO Box 30, FI-00271 Helsinki, Finland

e-mail: klas.winell@conmedic.fi
Conclusions/interpretation Despite the very large increase in the prevalence of diabetes, the PAF of the first CVD events due to diabetes decreased in women and increased only slightly in men.

Keywords Acute coronary syndrome $\cdot$ Cardiac complications $\cdot$ Cardiovascular event $\cdot$ Diabetes . Epidemiology · Macrovascular complications · Population attributable fraction $\cdot$ Stroke
Abbreviations
ACS Acute coronary syndrome
CVD Cardiovascular disease
ICD International Classification of Diseases
PAF Population attributable fraction

\section{Introduction}

Decreasing trends in cardiovascular event rates have been described in the general population in many countries from the 1980s to the 1990s, with some levelling off towards the new millennium [1-4]. On the other hand, the prevalence of diabetes is rapidly increasing in most countries [5], and it is known that diabetes approximately doubles the risk of cardiovascular disease (CVD) [6]. This may again lead to an increase in the number of CVD events.

The treatment and control of CVD risk factors, such as high blood cholesterol and high blood pressure, have improved among persons both with and without diabetes $[7,8]$, but other risk factors known to worsen the metabolic control of individuals with diabetes, such as BMI and abdominal obesity, are increasing [9]. The net effect of these conflicting developments on the population attributable 
fraction (PAF) of CVD events due to diabetes is not known. The large INTERHEART study estimated that $10 \%$ of myocardial infarctions in different countries were due to diabetes [10]. The recently published INTERSTROKE Study estimated that $7.9 \%$ of ischaemic strokes were caused by diabetes [11]. Both INTERHEART and INTERSTROKE were cross-sectional case-control studies, which did not assess longitudinal trends. There is some evidence that the CVD risk in the diabetic population is decreasing $[12,13]$ and also some evidence that the PAF of CVD due to diabetes may be increasing [14].

Mortality from CVD has been very high in Finland, but a steady decline in the mortality rate has taken place since the early 1970s [15]. The incidence of diabetes, known to be a major risk factor for acute coronary syndrome (ACS) and stroke, is rapidly increasing in Finland [16]. These two simultaneous phenomena can result in increasing CVD mortality and morbidity attributable to diabetes. Our aim was to examine whether the PAF for the first ACS, the first ischaemic stroke and the first CVD event (either ACS or stroke) in patients with diabetes had changed from 1992 to 2002 in Finland. We hypothesised that the PAFs of these adverse events due to diabetes would be increasing.

\section{Methods}

This study was accomplished by using data from four Finnish national registers: The National Hospital Discharge Register, the register of all medicines reimbursed in Finland, the register of patients with special reimbursement for certain diseases such as diabetes, and the Causes-ofDeath Register, covering the total population of 5.3 million people. The personal identification code made it possible to link the personal records across different registers. We used the reimbursement registers and the Hospital Discharge Register to identify all those treated for diabetes, and the Hospital Discharge Register and the Causes-of-Death register to identify all cases of ACS and stroke. The National Cardiovascular Disease Register, which is based on record linkage of the cardiovascular sections of the National Hospital Discharge Register and the National Causes-of-Death Register, was used to identify all ACS and ischaemic stroke events in both diabetic and non-diabetic individuals. The National Cardiovascular Disease Register has been described in detail [17-19]. A detailed description of the study register is available [20]. The study has been approved by the Ethical Committee of the National Institute for Health and Welfare.

Identification of ACS and ischaemic stroke International Classification of Diseases (ICD) ICD-9 (www.icd9data. com/2007/Volume1/240-279/250-259/250/default.htm) codes 410, 4110 and ICD-10 (www.who.int/classifications/ icd/en/) codes I20.0, I21 and I22 were used for identifying cases of non-fatal ACS in the National Hospital Discharge Register. ICD-9 codes 433-434 and 436 (excluding codes 4330X, 4331X, 4339X and 4349X) and ICD-10 codes I63I64 (excluding code I63.6) were used for identification of non-fatal ischaemic stroke. Fatal cases of ACS were identified with ICD-9 codes 410-414 and 798 and ICD10 codes I20-I25, I46, R96 and R98 in the Causes-ofDeath Register when used as the underlying or direct cause of death. The wider range of ICD codes was used for fatal events to fully capture out-of-hospital coronary deaths, for which clinical information may remain limited, despite the fact that an autopsy is often performed for such cases in Finland. Of the causes contributing to death, we included ICD-9 code 410 and ICD-10 codes I21-I22. Fatal cases of ischaemic stroke were identified on the basis of the same ICD-9 and ICD-10 codes as explained above for the non-fatal cases when used as the underlying, direct or contributing cause of death in the National Causes-of-Death Register.

A 4-year period without ACS or ischaemic stroke was used as a rule for both a fatal and a non-fatal first event; this was because the Hospital Discharge Register data went back to 1988. We kept the same 4-year rule throughout the study. In addition, the same 4-year rule was applied to patients who had had an ACS or stroke before they developed diabetes.

Analysis The number of patients with diabetes, the number of first ACS events, the number of first ischaemic strokes and the PAF due to diabetes were calculated for each year separately for men and women. We included in the study all patients in the age range $25-80$ years for each year. All calculations were made by full calendar years; in other words, if diabetes was diagnosed after the CVD event during the same calendar year, the event was considered to be a diabetic event. Annual population counts were obtained from the National Population Information System. The PAF due to diabetes was also counted by combining the figures for both cardiovascular events, i.e. either the first ACS or the first ischaemic stroke for an individual with diabetes.

We calculated the annual PAF (and 95\% CI) of the first ACS and first ischaemic stroke due to diabetes according to the formula $\mathrm{PAF}=(P \mathrm{e} \times(\mathrm{RR}-1) /$ $(1+(P \mathrm{e} \times(\mathrm{RR}-1))) \times 100$, where $P \mathrm{e}$ is the estimate of population exposure [21]. Trends for PAF were estimated by using log-linear regression models with the year as an independent variable. We also analysed whether PAF differed by the age group $(25-54,55-64$ and $65-$ 80 years old) in both sexes. The statistical analyses were carried out using SAS software version 9.1.3 (SAS, Chicago, IL, USA). 
Table 1 The crude prevalence of diabetes (per 100,000 inhabitants) among men and women aged 25-80 years and the rates of first ACS, first ischaemic stroke or first CVD event (either first ACS or first stroke) in diabetic patients (per 100,000 individuals with diabetes) aged 25-80 years in Finland in 1992, 1997 and 2002

\begin{tabular}{|c|c|c|c|c|c|c|c|c|}
\hline \multirow[t]{2}{*}{ Year } & \multicolumn{2}{|c|}{ Prevalence of diabetes } & \multicolumn{2}{|c|}{ Rate of the first ACS event } & \multicolumn{2}{|c|}{ Rate of the first stroke event } & \multicolumn{2}{|c|}{ Rate of the first CVD event } \\
\hline & Men & Women & Men & Women & Men & Women & Men & Women \\
\hline 1992 & 2,314 & 2,474 & 3,755 & 3,175 & 1,799 & 1,977 & 4,866 & 4,493 \\
\hline 1997 & 2,846 & 2,579 & 3,193 & 2,637 & 1,597 & 1,675 & 4,245 & 3,812 \\
\hline 2002 & 4,078 & 3,239 & 2,512 & 1,938 & 1,139 & 1,004 & 3,183 & 2,562 \\
\hline
\end{tabular}

\section{Results}

We identified a rapidly growing number of persons aged 25-80 years with diabetes in Finland over the years 19922002. The number of men treated for diabetes each year almost doubled during the study period, from 37,073 to 69,158 between 1992 and 2002. Among women, the increase was from 42,485 to 57,372 . Despite the increasing number of diabetic individuals, the annual number of first ACS events among persons with diabetes declined (among men from 10,211 in 1992 to 9,406 in 2002, and among women from 5,626 to 4,796), while the annual numbers of first ischaemic strokes stayed stable in men and declined in women. The annual rates of first ACS and ischaemic stroke events in diabetic persons (expressed per 100,000 individuals with diabetes) clearly declined (Table 1 and electronic supplementary material [ESM] Table 1).

The total number of individuals in the country with either a first ACS or first stroke event declined during the study period from 13,436 in 1992 to 12,678 in 2002 among men and from 8,658 to 7,521 among women. The corresponding annual rates of first ACS and ischaemic stroke events in the total population (expressed per 100,000 inhabitants) are presented in Table 2 and ESM Table 2, and show a clear decline. The ill-defined causes of death, R96, R98 and I46, were rare and constituted only $0.7 \%$ of fatal ACS cases. Of these, $98.6 \%$ were identified based on autopsy.

The PAF of the first ACS due to diabetes showed a diverging trend for men and women. Among men PAF increased from $11.6 \%(95 \%$ CI $10.9 \%, 12.3 \%)$ to $15.0 \%$ $(95 \%$ CI $14.2 \%, 15.8 \%)$ ( $p$ for trend $<0.0001)$ from 1992 to 2002, whereas among women PAF decreased nonsignificantly from $22.0 \%$ (95\% CI $20.9 \%, 23.2 \%)$ to $20.6 \%$ (95\% CI 19.4\%, 21.8\%) ( $p$ for trend 0.102 ; Table 3 and ESM Table 3). The trends in PAF were significantly different between the sexes $(p<0.001$ for the interaction year $\times$ sex).

A similar trend, but with a different strength of development, was found for the PAF of the first ischaemic stroke due to diabetes. Men had a PAF that increased from 14.8\% (95\% CI 13.6\%, 16.0\%) in 1992 to $16.0 \%(95 \%$ CI $14.7 \%, 17.3 \%)$ in 2002 ( $p$ for trend $0.065)$, whereas the PAF for women decreased from $21.5 \%$ (95\% CI $20.1 \%, 22.9 \%)$ to $15.1 \%$ (95\% CI $13.7 \%, 16.4 \%)$ ( $p$ for trend $<0.001$; Table 3 and ESM Table 3). Again, the trends in PAF were significantly different between the sexes $(p<0.001$ for the year $\times$ sex interaction).

The PAF of the first ACS remained at higher level for women than men, but the PAF of the first ischaemic stroke declined during the study period to a lower level among women than among men.

Analyses of the PAF of the first CVD event (either ACS or stroke) due to diabetes showed an increasing trend among men from $11.4 \%(95 \%$ CI $10.8 \%, 12.0 \%)$ to $13.8 \%(95 \%$ CI $13.2 \%, 14.5 \%$ ) ( $p$ for trend $<0.0001$ ), and a decreasing trend among women from $20.1 \%(95 \% \mathrm{CI}$ $19.2 \%, 21.0 \%)$ to $16.9 \%(95 \%$ CI $15.9 \%, 17.8 \%)$ ( $p$ for trend 0.0005) (Table 3 and ESM Table 3). These trends

Table 2 The rates of first ACS, first ischaemic stroke or first CVD event (either first ACS or first stroke event) in the total population of Finland in men and women per 100,000 inhabitants aged 25-80 years in 1992, 1997 and 2002

\begin{tabular}{|c|c|c|c|c|c|c|}
\hline \multirow[t]{2}{*}{ Year } & \multicolumn{2}{|c|}{ Rate of the first ACS event } & \multicolumn{2}{|c|}{ Rate of the first stroke event } & \multicolumn{2}{|c|}{ Rate of the first CVD event } \\
\hline & Men & Women & Men & Women & Men & Women \\
\hline 1992 & 637 & 328 & 248 & 208 & 839 & 504 \\
\hline 1997 & 567 & 291 & 253 & 195 & 775 & 456 \\
\hline 2002 & 555 & 271 & 239 & 183 & 748 & 425 \\
\hline
\end{tabular}


Table 3 PAF and trends in PAF of first ACS, first ischaemic stroke or first CVD event (either ACS or stroke) due to diabetes among men and women aged 25-80 years in Finland in 1992, 1997 and 2002

\begin{tabular}{|c|c|c|c|c|c|c|}
\hline \multirow[t]{2}{*}{ Year } & \multicolumn{2}{|c|}{ PAF $(95 \% \mathrm{CI})$ for the first ACS } & \multicolumn{2}{|c|}{ PAF $(95 \% \mathrm{CI})$ for the first stroke } & \multicolumn{2}{|c|}{ PAF $(95 \% \mathrm{CI})$ for the first CVD event } \\
\hline & Men & Women & Men & Women & Men & Women \\
\hline 1992 & $11.6(10.9,12.3)$ & $22.0(20.9,23.2)$ & $14.8(13.6,16.0)$ & $21.5(20.1,22.9)$ & $11.4(10.8,12.0)$ & $20.1(19.2,21.0)$ \\
\hline 1997 & $13.6(12.8,14.3)$ & $21.3(20.1,22.5)$ & $15.6(14.4,16.8)$ & $20.1(18.7,21.6)$ & $13.1(12.5,13.8)$ & $19.5(18.6,20.4)$ \\
\hline 2002 & $15.0(14.2,15.8)$ & $20.6(19.4,21.8)$ & $16.0(14.7,17.3)$ & $15.1(13.7,16.4)$ & $13.8(13.2,14.5)$ & $16.9(15.9,17.8)$ \\
\hline Trend $^{\mathrm{a}}$ & $p<0.0001$ & $p=0.102$ & $p=0.065$ & $p<0.001$ & $p<0.0001$ & $p=0.0005$ \\
\hline
\end{tabular}

${ }^{a} p$ value for the linear trend during the study period

were significantly different between the sexes $(p<0.001$ for the interaction year $\times$ sex). PAF increased clearly by age in women, whereas in men the increase was modest (Table 4).

\section{Discussion}

Despite the considerable increase in the prevalence of diabetes, the PAF of the first CVD event decreased in women and increased only slightly in men. This suggests that the risk of CVD in diabetic individuals has markedly decreased. Nevertheless, the contribution of diabetes to the burden of the first CVD events remained considerable and, in spite of the different developments between the sexes, stayed clearly higher in women than in men. In the last year of our study, the PAF of the first CVD event due to diabetes was $13.8 \%$ in men and $16.9 \%$ in women. The PAF of the first stroke reached the same level among women as among men, $15.1 \%$ vs $16.0 \%$ in 2002 . If this development continues in the same direction, the difference between the sexes in the PAF of the first CVD event may disappear during the next 10 years.

Very few studies have recently analysed the PAF of CVD due to diabetes. Our findings, showing an increasing PAF of the first CVD event due to diabetes in men, was in line with the report from the Framingham Heart Study [14]. In the Framingham Heart Study, the researchers followed a narrower age group of individuals (45-64 years) than we did. They examined all CVD events, not only the first ones, including also transient ischaemic attacks, claudication and heart failure. The study showed no change in the PAF for women, whereas we found a significant reduction. In our study, the differences between the sexes in terms of having a first ACS or stroke event could be a result of differences in the intensity of treatment of risk factors or different responses to treatment.

The decreasing trend was significant for the PAF of the first ischaemic stroke in women, but not significant for the first ACS. The increasing trend was significant for the PAF of the first ACS in men, but not significant for the first

Table 4 PAF of first ACS, first ischaemic stroke and first event of either of these (CVD) with 95\% CI by age group in men and women in 1992 and 2002 in Finland

\begin{tabular}{|c|c|c|c|c|c|}
\hline \multirow[t]{2}{*}{ Disease } & \multirow[t]{2}{*}{ Age group (years) } & \multicolumn{2}{|l|}{ Men } & \multicolumn{2}{|l|}{ Women } \\
\hline & & 1992 & 2002 & 1992 & 2002 \\
\hline \multirow[t]{3}{*}{ ACS } & $25-54$ & $7.6(6.2,8.9)$ & $10.8(9.1,12.5)$ & $11.2(7.3,14.9)$ & $6.3(3.4,9.1)$ \\
\hline & $55-64$ & $7.9(6.6,9.2)$ & $10.8(9.5,12.5)$ & $16.1(13.1,19.0)$ & $13.4(10.2,16.4)$ \\
\hline & $65-80$ & $8.3(7.2,9.3)$ & $11.2(7.3,14.9)$ & $18.4(17.0,19.8)$ & $17.4(15.9,18.9)$ \\
\hline \multirow[t]{3}{*}{ Stroke } & $25-54$ & $9.0(6.4,11.5)$ & $8.1(5.4,10.7)$ & $5.2(2.0,8.4)$ & $4.0(1.2,6.7)$ \\
\hline & $55-64$ & $12.9(10.3,15.4)$ & $13.2(10.4,15.9)$ & $16.5(12.6,20.3)$ & $7.9(4.6,11.0)$ \\
\hline & $65-80$ & $10.9(9.3,12.5)$ & $10.3(8.5,12.1)$ & $18.1(16.4,19.8)$ & $11.3(9.5,13.0)$ \\
\hline \multirow[t]{3}{*}{ CVD } & $25-54$ & $7.1(6.0,8.3)$ & $6.4(5.2,7.6)$ & $8.1(5.6,10.7)$ & $4.4(2.5,6.3)$ \\
\hline & $55-64$ & $7.8(6.6,8.9)$ & $10.4(9.0,11.9)$ & $14.9(12.6,17.2)$ & $10.1(7.9,12.3)$ \\
\hline & $65-80$ & $8.0(7.1,8.9)$ & $8.9(7.9,10.0)$ & $16.3(15.2,17.4)$ & $13.3(12.1,14.5)$ \\
\hline
\end{tabular}


ischaemic stroke. The diverging development for men and women is probably mainly due to the greater increase in obesity among men than women [15], which has increased the number of cases of diabetes more in men than in women. Interestingly, the PAF of the first CVD event due to diabetes decreased among women, although the number of women with diabetes increased. This suggests that the total CVD risk has decreased among diabetic women, or that the share of other risk factors may have increased. It is known that the prevalence of smoking increased among women during the study period, and this may have reduced the PAF due to diabetes. The distinct increase in PAF by age in women is probably due to the increase in the prevalence of diabetes with increasing age, but it is not clear why PAF is not increasing in men in the same manner.

The strengths of our study are the long study period, the large number of diabetic patients and the fact that we were able to cover the total population of the country, all diabetic patients treated with medications and all CVD events, including out-of-hospital sudden coronary deaths. The main limitation was that we could not identify diabetic individuals treated with diet alone, which may make our estimates of PAF somewhat conservative. Another limitation was that our study setting did not allow us to take other risk factors into account in PAF calculations to better control for confounding. The 4-year event-free rule may not have excluded all people with a prior ACS or stroke, and improvements in medical care may mean that the proportion of recurrent events mistakenly identified as incident events has increased over time.

In conclusion, the PAF of the first CVD event due to diabetes was high and increased slightly among men. Somewhat surprisingly, the PAF decreased among women, despite the increasing number of women with diabetes. This suggests that the risk of a CVD event has declined considerably among women with diabetes. Nevertheless, the PAF of the first CVD event due to diabetes remained higher among women than men.

Acknowledgements This study has been supported by grants from the Finnish Diabetes Research Foundation (K. Winell) and the Finnish Foundation for Cardiovascular Research (V. Salomaa).

Contribution statement $\mathrm{KW}$ was the principal author, contributed to data acquisition, study design and interpretation of the results, and drafted the manuscript text. AP carried out the statistical analyses and contributed to the interpretation of the results and revision of the manuscript. MN contributed to data acquisition, study design and revision of the text. AR contributed to data acquisition, study design and revision of the text. VS led the research group and contributed to the study design, data analyses and interpretation as well as the revision of the manuscript. All authors have approved the final text.

Duality of interest The authors declare that there is no duality of interest associated with this manuscript.

\section{References}

1. Truelsen $\mathrm{T}$, Mähönen $\mathrm{M}$, Tolonen $\mathrm{H}$, Asplund $\mathrm{K}$, Bonita $\mathrm{R}$, Vanuzzo D, WHO MONICA Project (2003) Trends in stroke and coronary heart disease in the WHO MONICA Project. Stroke 34:1346-1352

2. Ferrario M, Cesana G, Vanuzzo D et al (2001) Surveillance of ischaemic heart disease: results from the Italian MONICA populations. Int J Epidemiol 30(Suppl 1):S23-S29

3. Lampe FC, Morris RW, Whincup PH, Walker M, Ebrahim S, Shaper AG (2001) Is the prevalence of coronary heart disease falling in British men? Heart 86:499-505

4. O'Flaherty M, Ford E, Allender S, Scarborough P, Capewell S (2008) Coronary heart disease trends in England and Wales from 1984 to 2004: concealed levelling of mortality rates among young adults. Heart 94:178-181

5. International Diabetes Federation (2009) IDF Diabetes Atlas, 4th edn. www.diabetesatlas.org/content/diabetes-and-impaired-glucosetolerance. Accessed 15 April 2010

6. The Emerging Risk Factors Collaboration (2010) Diabetes mellitus, fasting blood glucose concentration, and risk of vascular disease: a collaborative meta-analysis of 102 prospective studies. Lancet 375:2215-2222

7. Preis SR, Pencina MJ, Hwang SJ et al (2009) Trends in cardiovascular disease risk factors in individuals with and without diabetes mellitus in the Framingham Heart Study. Circulation 120:212-220

8. Suh DC, Kim CM, Choi IS, Plauschinat CA, Barone JA (2009) Trends in blood pressure control and treatment among type 2 diabetes with comorbid hypertension in the United States: 19882004. J Hypertens 27:1908-1916

9. Kramer H, Cao G, Dugas L, Luke A, Cooper R, Durazo-Arvizu R (2010) Increasing BMI and waist circumference and prevalence of obesity among adults with type 2 diabetes: the National Health and Nutrition Examination Surveys. J Diabetes Complications 24:368-374

10. Yusuf S, Hawken S, Ounpuu S, INTERHEART Study Investigators et al (2004) Effect of potentially modifiable risk factors associated with myocardial infarction in 52 countries (the INTERHEART study): case-control study. Lancet 364:937-952

11. O'Donnell MJ, Xavier D, Liu L, on behalf of the INTERSTROKE investigators et al (2010) Risk factors for ischaemic and intracerebral haemorrhagic stroke in 22 countries (the INTERSTROKE study): a case-control study. Lancet 376:112-123

12. Fox CS, Coady S, Sorlie PD et al (2004) Trends in cardiovascular complications of diabetes. JAMA 292:2495-2499

13. Booth GL, Kapral MK, Fung K, Tu JV (2006) Recent trends in cardiovascular complications among men and women with and without diabetes. Diabetes Care 29:32-37

14. Fox CS, Coady S, Sorlie PD et al (2007) Increasing cardiovascular disease burden due to diabetes mellitus: the Framingham Heart Study. Circulation 115:1544-1550

15. Vartiainen E, Laatikainen T, Peltonen M et al (2010) Thirty-fiveyear trends in cardiovascular risk factors in Finland. Int $\mathrm{J}$ Epidemiol 39:504-518

16. Sund R, Koski S, Fin DM II (2009) On the register-based measurement of the prevalence and incidence of diabetes and its long-term complications. A technical report. Tampere: Finnish Diabetes Association [Report on line]. http://www.diabetes.fi/files/ 1167/DehkoFinDM_Raportti_ENG.pdf. Accessed 12 July 2011

17. Mähönen M, Salomaa V, Keskimäki I, Moltchanov V (2000) The feasibility of routine mortality and morbidity register data linkage to study the occurrence of acute coronary heart disease events in Finland. The Finnish Cardiovascular Diseases Registers (CVDR) Project. Eur J Epidemiol 16:701-711 
18. Pajunen P, Pääkkönen R, Juolevi A et al (2004) Trends in fatal and non-fatal coronary heart disease events in Finland during 1991-2001. Scand Cardiovasc J 38:340-344

19. Pajunen $P$, Pääkkönen R, Hämäläinen $H$ et al (2005) Trends in fatal and nonfatal strokes among persons aged 35 to $>$ or $=85$ years during 1991-2002 in Finland. Stroke 36:244-248
20. Winell K, Pääkkönen R, Pietilä A, Niemi M, Reunanen A, Salomaa V (2010) Case fatality rates after first acute coronary syndrome in persons treated for type 2 diabetes show an improving trend. Diabetologia 53:472-480

21. Lachin JM (2000) Biostatistical methods. The assessment of relative risks. Wiley, New York 